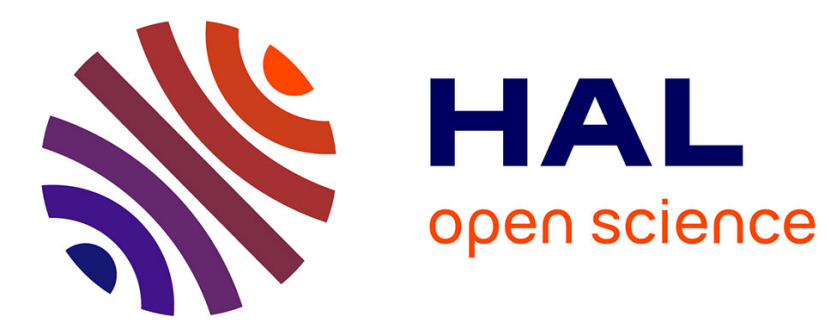

\title{
Stochastic model for differential Mueller matrix of stationary and non-stationary turbid media
}

\author{
J-M Charbois, V Devlaminck
}

\section{To cite this version:}

J-M Charbois, V Devlaminck. Stochastic model for differential Mueller matrix of stationary and nonstationary turbid media. Journal of the Optical Society of America. A, Optics and image science, 2016, 33 (12), pp.2414-2424. 10.1364/JOSAA.33.002414 . hal-01399899

\section{HAL Id: hal-01399899 \\ https://hal.science/hal-01399899}

Submitted on 21 Nov 2016

HAL is a multi-disciplinary open access archive for the deposit and dissemination of scientific research documents, whether they are published or not. The documents may come from teaching and research institutions in France or abroad, or from public or private research centers.
L'archive ouverte pluridisciplinaire HAL, est destinée au dépôt et à la diffusion de documents scientifiques de niveau recherche, publiés ou non, émanant des établissements d'enseignement et de recherche français ou étrangers, des laboratoires publics ou privés. 


\title{
Stochastic model for differential Mueller matrix of stationary and non-stationary turbid media
}

\author{
J.M. Charbois, V. DeVlaminck* \\ Université de Lille, CRIStAL, UMR 9189, 59650 Villeneuve d'Ascq, France
}

\section{INTRODUCTION}

The differential Mueller matrix formalism takes into account the continuous variation of the optical properties of a medium according to the thickness $\mathrm{z}$ of the traversed medium. It relates the Mueller matrix $\mathbf{M}(z)$ at a distance $z$ into the medium to its spatial derivative along the light propagation direction by:

$$
\frac{d \mathbf{M}(z)}{d z}=\mathbf{m}(z) \mathbf{M}(z)
$$

where $\mathbf{m}(z)$ is termed differential Mueller matrix. For a non depolarizing Mueller matrix, $\mathbf{m}(z)$ is a deterministic matrix completely determined [1] by its decomposition on the basis of the six generators $\mathbf{G}_{1-6}$ of $S O(3,1)$ (these generators are described in Appendix A of [2]) weighted by $p_{1-6}(z)$ coefficients. ( $p_{1-3}$ for linear, $\pm 45^{\circ}$ and circular birefringence and $p_{4-6}$ for dichroism) and $\mathbf{G}_{0}$ the identity matrix weighted by the differential isotropic absorption $p_{0}(z)$ :

$$
\mathbf{m}_{\mathrm{ND}}(z)=\sum_{i=0}^{6} p_{i}(z) \mathbf{G}_{i}
$$

Different authors ([2,3] and [4] in an alternative form) proposed to consider the differential Mueller matrix associated to a depolarizing medium as a matrix of random processes generated by the fluctuations of these birefringence and dichroïsm components. Eq. (1) is then a stochastic differential equation and we are actually only interested in the average value of the solution of Eq. (1) since $\mathbf{M}(z)$ is then seen as an average value of nondepolarizing Mueller matrices $\mathbf{M}_{\mathrm{ND}}(z)$ associated to their differential matrices $\mathbf{m}_{\mathrm{ND}}(z)$ but random.

Recently Agarwal \& al. [5] analyzed the depolarization of a turbid medium consisting of submicron scattering particles from the differential Mueller matrix point of view. The turbid media under study were assemblies of rutile (TiO2) submicron particles homogeneously embedded in a PVC-based transparent host material. Their paper contains two main results: depolarization evolves parabolically with the thickness of the medium traveled by light and the standard deviations of the elementary polarization properties vary linearly with the concentration of scatterers. On another side, from a point of view which considers thickness of the medium on a much longer scale than the scale determined by the process correlation distance, we demonstrated [3] that this hypothesis of finite horizon correlation leads to have a linear variation on a large scale. Given the nature of the medium and concentrations used in the experiment conducted in [5], the second operating region is not explicitly visible (although partly already present) in the results reported by these authors.

The goals of this paper are firstly to experimentally validate the demonstration previously presented in [3] and also to show that the results in [5] correspond to a particular case of behavior that can be generalized to describe a much broader set of turbid media. More specifically, experimental results obtained in turbid media consisting of particles suspended in liquids, clearly show that the depolarization does not necessarily evolve parabolically with the thickness of the medium traveled by light. The general model we proposed in [3], perfectly describes these new experimental results or those presented in [5]. Finally a stochastic solution is proposed to model the phenomenon. It perfectly describes all these different experimental results and allows to analyze the different regimes in terms of correlation of the fluctuations that are responsible for depolarization.

These contributions are of significant practical interest because considerable attention has been recently focused on the studies of polarized light propagation in turbid media for industrial, medical or communication technology applications [6-10]

\section{EXPERIMENTAL RESULTS}

We measured the Mueller matrix of a variable-length sample obtained by firstly mixing full cream or half cream milk with water and secondly an anise-flavored spirit (called pastis) with water. The light is scattered, either by the casein pellets in the case of milk, or by precipitation of the anethole in the case of pastis (anethole is only slightly soluble in water but exhibits high solubility in ethanol).

The full cream milk diluted in water in a ratio $0.048,0.095$ and 0.167 (half cream milk in a ration 0.286 and 0.429 and the pastis in a ratio 0.231 and 0.276 respectively) is placed into a polarimetrically neutral glass container between the generator and the analyzer of a Mueller polarimeter. The concentration in the water is kept constant during measurement. The thickness of the medium traveled by light is set by the liquid level. The illumination consists of a $627 \mathrm{~nm}$ LED source with a spectral width of $20 \mathrm{~nm}$. A set of lenses and a diffuser are used to collect the light and control the divergence of the probing beam. A diaphragm placed at the mounting input (not shown) adjusts the sample surface which is illuminated. Another diaphragm placed at the mounting output (not shown) adjusts the surface of light collected by the photodiode. The entire sample surface has a 
diameter of $3 \mathrm{~cm}$. The light is collected by a standard Si photodiode with a diameter of $9 \mathrm{~mm}$. The sample and the photodiode are separated by 470 $\mathrm{mm}$. The incident angle of the sample outgoing rays collected by the photodiode (without output diaphragm), therefore ranges from $0^{\circ}$ to $0.6^{\circ}$ for a secondary source placed on the optical axis, and from $1.2^{\circ}$ to $2.3^{\circ}$ for a secondary source placed on the edges of the sample.

The polarimeter state generator (PSG) and analyzer (PSA) are built with rotating wave plates and linear polarizers. For each position of the polarimeter, the measured intensity is the result of an average of 1000 acquisitions. A schematic presentation of the polarimeter is given Fig. 1. For presentation purposes, the diagram is shown in a horizontal position while the actual mounting is vertical.

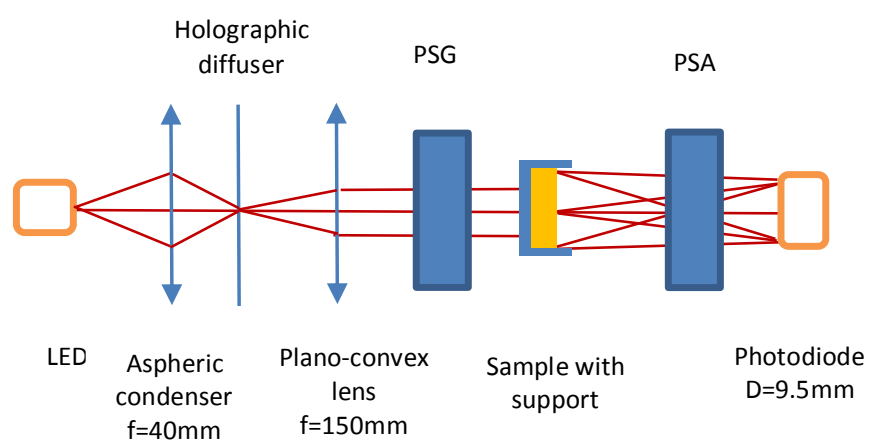

Fig. 1. Schematic presentation of the Mueller matrix polarimeter

The measured Mueller matrix $\mathbf{M}$ associated to this forward scattering medium, is a diagonal one with the following shape (normalized by mo0)

$$
\mathbf{M}=\operatorname{diag}\left(1 \quad k_{1} \quad k_{2} \quad k_{3}\right)
$$

with possibly $k_{2}=k_{1}$ if a rotationally invariance property with respect to the propagation axis [11] is present. The matrix logarithm of $\mathbf{M}$ is then a diagonal matrix with three non-null entries $\alpha_{\mathrm{i}}=\log \left(k_{\mathrm{i}}\right)$. For each of the 1000 acquisitions, a Mueller matrix is calculated. 1000 values of $\alpha_{\mathrm{I}}$ the logarithmic depolarization are therefore obtained from which mean value and standard deviation are estimated. This is repeated for each measurement point on the curves. In all the cases of dilutions, the estimated standard deviation from the 1000 acquisitions takes values less than $1 / 100$ of the average whatever the measured thickness of liquid.

Evolution of the logarithmic depolarizations $\alpha_{1}$ with $z$ the thickness of the medium traveled by light is presented in Fig. 2 for the three dilution values of full cream milk. The solid line fitting the experimental values is derived from the model developed in the following section. As in [5], the results for $\alpha_{3}$ are very similar and not presented in order not to overload the figure.

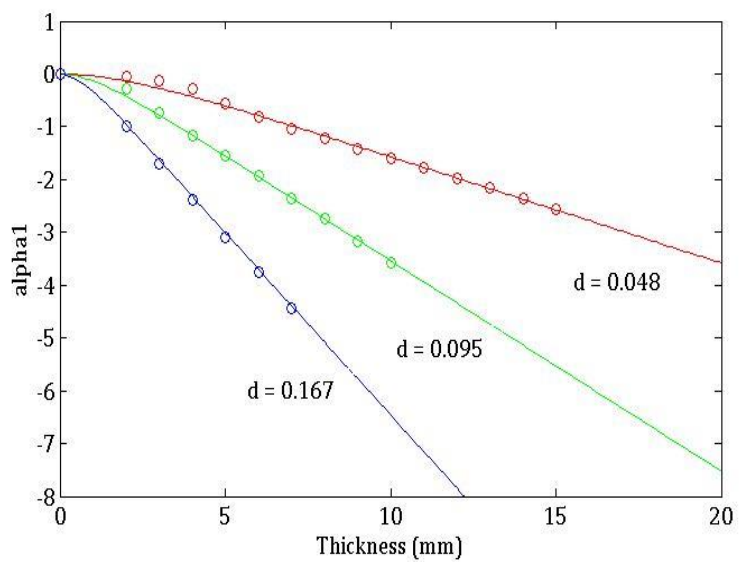

Fig. 2. Evolution of the logarithmic depolarizations $\alpha_{1}$ with $z$ the thickness of medium traveled by light for three dilutions $d$ of full cream milk. o are experimental values for the three $d$ values. The solid line is derived from Eq. (13).

Same measurements are presented Fig. 3 for the two dilution values of half cream milk. The results for $\alpha_{3}$ are very similar and not presented. Eventually for the diluted pastis the evolution of both the logarithmic depolarizations $\alpha_{1}$ and $\alpha_{3}$ that are significantly different are shown Fig. 4 and Fig. 5 respectively. 


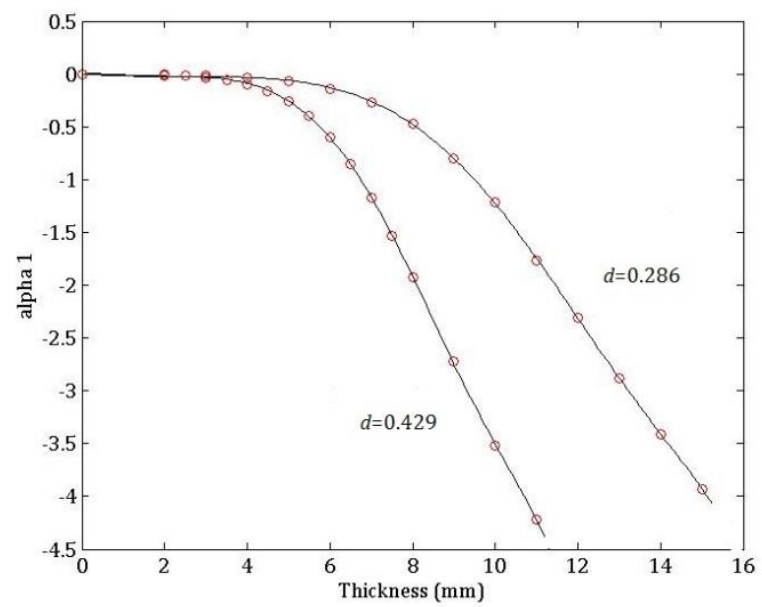

Fig. 3. Evolution of the logarithmic depolarizations $\alpha_{1}$ with $z$ the thickness of medium traveled by light for two dilutions $d$ of half cream milk. o are experimental values for both the $d$ values. The solid line is derived from Eq. (13).

It is possible to make various comments from these measurement results:

- In all cases, except for the first few points, variations are clearly linear when zis large enough.

- Even for the first points of measurement (small z values) the logarithmic depolarization does not necessarily evolve parabolically with the thickness of the medium. Some illustrations are presented in section 4 showing the dependency law observed with the measurement setups used. For the same medium, the different $\alpha_{\mathrm{l}}$ logarithmic depolarizations may have quite different behaviors as a function of $\mathrm{z}$ (See Section 3 for explanation of these results).

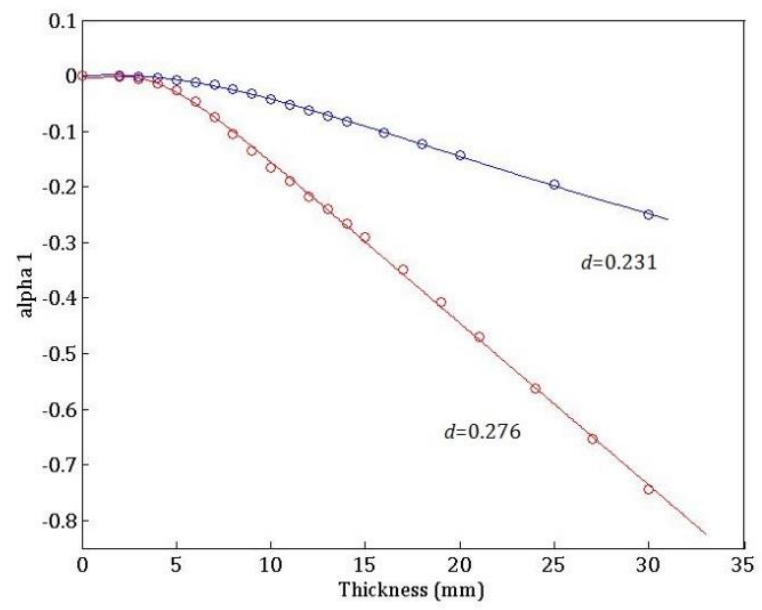

Fig. 4. Evolution of the logarithmic depolarizations $\alpha_{1}$ with $z$ the thickness of medium traveled by light for two dilutions $d$ of pastis. $o$ are experimental values for both the $d$ values. The solid line is derived from Eq. (13).

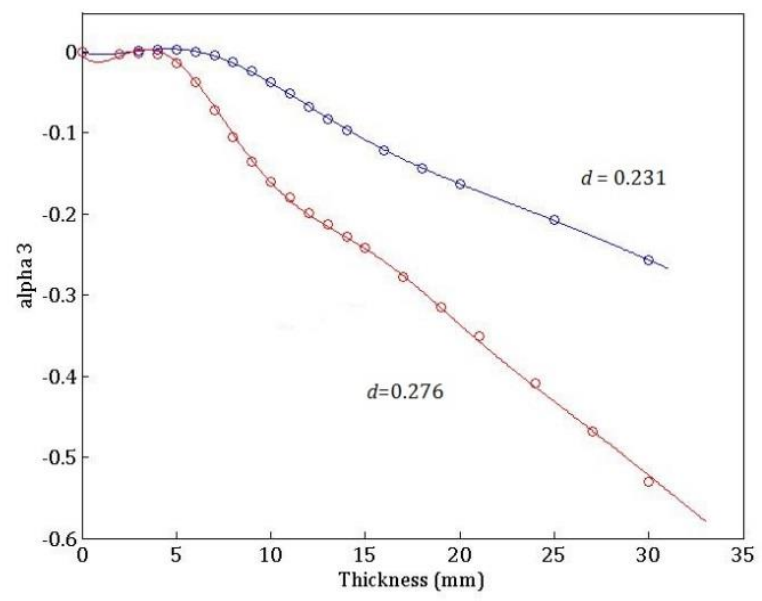


Fig. 5. Evolution of the logarithmic depolarizations $\alpha_{3}$ with $z$ the thickness of medium traveled by light for two dilutions $d$ of pastis. o are experimental values for both the $d$ values. The solid line is derived from Eq. (24).

\section{STOCHASTIC MODEL}

\section{A. Scattering media}

The scattering media we investigated are typical examples of non-stationary samples [12]. The Brownian motion of suspended particles in a liquid induces non-stationary temporal fluctuations of the optical properties of the medium. Temporal average of such fluctuations cause depolarization effect that can be considered as intrinsic to the medium but also related to the time average performed during the measurement. It is worth noticing that this result holds even for a single particle. In the case where the number of particles that act is now assumed sufficiently large for them to be distributed continuously along $z$ the thickness of medium (not to be confused with the optical path length) and a particle can no longer be distinguished from another, the optical properties become non-stationary processes with regard to $z$ and $t$ quantities. At the scale of the sensor integration time used (a few $m s$ ) we are obviously operating in static and not dynamic context. The transient regime is supposed to be completed and it isno longer necessary to take time into account in the equations. From the point of view of the evolution according to the "time" variable, the process can be considered stationary. We are therefore justified to consider the optical properties only as non-stationary processes of $z$ variable, meaning that its statistical moments depends on $z_{0}$ the origin point. It is worth noticing that no spatial averaging is necessary in this case.

Now consider the case of solid media as investigated in [5]. For a solid medium with static scatterers such temporal fluctuations do not exist. Along the optical path traveled by a light wave, each interaction with a particle modifies the wave polarization state, but does not depolarize. Depolarization only occurs in the presence of optical path averaging process performed by the detection scheme. However each optical path can be considered as one realization of a fluctuating process (the number of particles along the optical path is always assumed large enough that the particles are regarded as indistinguishable).

Under the hypothesis of ergodicity (and stationarity) on such processes, all the moments are equal to the statistical moments. These statistical moments are obtained by computing the moments on all the possible events of the $p(z)$ process. It is worth noticing that a spatial averaging is mandatory in this case.

So from a stochastic perspective, the case of solid media is the stationary version of the case of liquid media. From a practical point of view, the stationary solution may be regarded as the limit when $z_{0}=-\infty$ of the non-stationary case.

\section{B. Stochastic model for pure scattering media}

The stochastic differential equation given Eq. (1) can be formally solved using the z-ordered operator introduced by Fox [13]:

$$
\mathbf{M}(\mathrm{z})=\left\langle\mathbf{M}_{\mathrm{ND}}(\mathrm{z})\right\rangle=\left\langle\underset{\leftarrow}{Z} \exp \left[\int_{0}^{\mathrm{z}} \mathbf{m}_{\mathrm{ND}}(s) d s\right]\right\rangle \mathbf{M}(0)
$$

where $Z$ is a space-ordering operator that causes the factors in a product to appear in the same sequence as in the medium of interest. This spaceordering operator is made necessary since the matrices $\mathbf{m}_{\mathrm{ND}}\left(Z_{1}\right)$ and $\mathbf{m}_{\mathrm{ND}}\left(Z_{2}\right)$ do not commute for $Z_{1} \neq Z_{2}$ in general. Such products actually appear when the explicit form of the exponential is achieved by using a power series expansion leading to an explicit determination of any order moment. It is well known $[13,14]$ that the evaluation of Eq. (4) is better suited by using the $z$-ordered cumulants. We have:

$$
\left\langle\underset{\leftarrow}{Z} \exp \left(\int_{0}^{\mathrm{z}} \mathbf{m}_{\mathrm{ND}}(s) d s\right)\right\rangle=\underset{\leftarrow}{Z} \exp \left(\sum_{n=1}^{+\infty} \int_{0}^{Z} \mathbf{c}^{(\mathrm{n})}(s) d s\right)
$$

where $\mathbf{c}^{(\mathrm{n})}$ stands for the cumulant of $n$ order. This latter equation describes the solution for any matrix $\mathbf{m}_{\mathrm{ND}}(\mathrm{z})$. With such an approach, the differential Mueller matrix $\mathbf{m}(z)$ can be decomposed into its non-depolarizing and depolarizing part verifying the Minkowski antisymmetric and symmetric property respectively as proposed by Ossikovski [15]. These two parts are then straightforwardly written from the differentstatistical moments of the $p_{\mathrm{i}}(\mathrm{z})$ processes (see Eqs. (31)-(32) in [3]).

In the very particular situation of diagonal Mueller matrices (pure depolarizer) simplifications are possible. In this case, the differential Mueller matrix is diagonal too meaning that the stochastic processes $p_{\mathrm{i}}(\mathrm{z})$ are independent [2]. If we combine this property with the assumption that all the $p_{\mathrm{i}}(\mathrm{z})$ processes have a null mean value - meaning that the non-depolarizing part of $\mathbf{m}(z)$ is reduced to null matrix (see [3]) which is consistent with the hypothesis of a pure depolarizing Mueller matrix, the only matrices that are to be considered in the calculation of cumulants are obtained by multiplying $\mathbf{G}_{\mathrm{i}}$ by repeatedly itself. This result shows firstly that we are no more in the presence of stochastic operators but scalar processes since all the matrix products are commutative in this case. The $Z$ space-ordering operator is then not necessary and Eq. (5) would reduce to an ordinary exponential. Multiplying $\mathbf{G}_{\mathbf{i}}$ by repeatedly itself leads alternatively to Minkowski antisymmetric and symmetric matrices. This means that $\mathbf{m}(z)$ should have a non-depolarizing part made by some $\mathbf{c}^{(\mathrm{n})}$ cumulants of order higher than 2 . However this is not observed experimentally in [5] or in our experiments.

From this, it is reasonable to expect that $\mathbf{m}(z)$ represents a Gaussian process. Indeed, it is well known that under the assumption of a Gaussian scalar process, all the cumulants of higher order than second cumulant are equal to zero. The model is therefore consistent with the experimental results obtained in [5] and in our experiments. It is worth noticing that this result is specific to diagonal $\mathbf{m}(z)$. For arbitrary $\mathbf{m}(z)$ matrix, even if it is assumed to be Gaussian, the non-commutativity of this matrix for different $z$ values, annihilates this classical scalar property [3].

It follows from all these remarks that in the case of diagonal matrices, $\mathbf{M}(z)$ can be obtained from an equation of the form:

$$
\mathbf{M}(z)=\exp \left[\sum_{i=0}^{6}\left(\mathbf{G}_{i} \mathbf{G}_{i} \int_{0}^{z} \int_{0}^{z}\left\langle p_{i}(u) p_{i}(v)\right\rangle d u d v\right)\right]
$$

Given the above observations and the experimental results reported in [5] and those presented Fig. 2 to Fig.5, the $p_{\mathrm{i}}(z)$ process for modeling the behavior of $\mathbf{M}(z)$ in the case of homogeneous turbid media, should therefore be Gaussian. For small $z$ values and stationary solution, $\log (\mathbf{M})$ should 
follow a parabolic law with the thickness of the medium traveled by light but a linear law on a large scale for both the stationary and non-stationary hypothesis.

From a microscopic scale each optical property $p$ evolves from a state $p(n)$ to a state $p(n+1)$ due to the action of the $n$th particle with an update formula given by:

$$
p(n+1)=a p(n)+B
$$

where $B$ stands for the random perturbation due to the $n$th fluctuating particles. Eq. (7) governs autoregressive order one processes. The continuous version of such discrete processes are known as Orstein-Uhlenbeck $(O U)$ processes [16]. $p(z)$ as a OUprocess solves the stochastic differential equation [17]:

$$
d p(z)=-a p(z) d z+\sigma d B(z)
$$

where $B$ stands for a Brownian process, $a$ is a parameter specifying how strongly the system reacts to perturbations and $\sigma$ is related to the noise amplitude.

The update formula for $p$ associated to this differential stochastic equation is given by:

$$
p(z+d z)=p(z)-a p(z) d z+\sigma N(z)(d z)^{1 / 2}
$$

Where $N(z)$ is a unit normal random variable with $N\left(z_{1}\right)$ statistically independent of $N\left(z_{2}\right)$ if $z_{1} \neq z_{2}$. According to Eq. (9), $a$ is a parameter defined per-unit-length (the dimension of a parameter $x$ is denoted by the symbol $[x]$ ) and $[\sigma]=\mathrm{L}^{-3 / 2}$ since $[\mathrm{p}]=\mathrm{L}^{-1}$ from Eq. (6). The statistical property of $O U$ processes are well established and can be summarized in their non-stationary version by the following properties: $p(z)$ is Gaussian process with mean value $E[p(z)]$ and covariance $\operatorname{Cov}\left[p\left(z_{1}\right) p\left(z_{2}\right)\right]$ (defined by $\operatorname{Cov}\left[p\left(z_{1}\right) p\left(z_{2}\right)\right]=E\left[\left(p\left(z_{1}\right)-\mathrm{E}\left(p\left(z_{1}\right)\right)\right)\left(p\left(z_{2}\right)-\mathrm{E}\left(p\left(z_{2}\right)\right)\right)\right]$ given by:

$$
\left\{\begin{array}{c}
E[p(z)]=x_{0} e^{-a\left(z-z_{0}\right)} \\
\operatorname{Cov}\left[p\left(z_{1}\right) p\left(z_{2}\right) / z_{0}\right]=\frac{\sigma^{2} e^{-a\left(z_{2}-z_{1}\right)}}{2 a}\left[1-e^{-2 a\left(z_{1}-z_{0}\right)}\right] \\
\left(z_{0} \leq z_{1} \leq z_{2}\right) \text { and } p\left(z_{0}\right)=x_{0}
\end{array}\right.
$$

From Eq. (6), the diagonal entries of $\log (\mathbf{M})$ can be considered as variance of $\Delta_{i}$ processes defined by:

$$
\Delta_{i}(z)=\Delta_{i}(0)+\int_{0}^{z} p(s) d s
$$

$p(z)$ is then the rate of change of the process $\Delta_{i}$ and that is this velocity that is influenced by the perturbations. We obviously have $\Delta_{i}(0)=0$ in the case that we consider since $p\left(z_{0}=0\right)=\mathrm{x}_{0}=0$. It means that the optical properties do not change before reaching the beginning of the sample which is assumed to be at $z_{0}=0$.

Note that the $p(z)$ process is Gaussian and therefore satisfies the first of the desired properties and $\Delta_{i}(z)$ is a Gaussian process because $O U$ is Gaussian.

An application of Brownian process properties [16-18] gives for the variance of $\Delta_{i}$ processes:

$$
\begin{aligned}
& \int_{0}^{z} \int_{0}^{z}\left\langle p_{i}(u) p_{i}(v)\right\rangle_{z_{0}} d u d v=\sigma_{\Delta_{i}}^{2}\left(z / z_{0}\right) \\
& =\frac{\sigma^{2}}{2 a^{3}}\left(2 a z-2-e^{2 a z_{0}}-e^{-2 a\left(z-z_{0}\right)}+2 e^{-a\left(z-2 z_{0}\right)}+2 e^{-a z}\right)
\end{aligned}
$$

Eq. (12) leads to the following solutions for the stationary $\left(\mathrm{z}_{0} \rightarrow-\infty\right)$ and non-stationary $\left(\mathrm{z}_{0}=0\right)$ cases:

$$
\left\{\begin{array}{c}
\lim _{z_{0} \rightarrow-\infty} \sigma_{\Delta_{i}}^{2}\left(z / z_{0}\right)=\frac{\sigma^{2}}{a^{3}}\left(a z-1+e^{-a z}\right) \\
\sigma_{\Delta_{i}}^{2}\left(z / z_{0}=0\right)=\frac{\sigma^{2}}{2 a^{3}}\left(2 a z-3-e^{-2 a z}+4 e^{-a z}\right)
\end{array}\right.
$$

For small and large values of $z$, Eq. (13) can be rewritten as:

$$
\sigma_{\Delta_{i}}^{2}(z)=\left\{\begin{array}{c}
\frac{\sigma^{2}}{a^{2}} z \text { if } a z \text { is large } \\
\frac{\sigma^{2}}{2 a} z^{2} \text { with } \mathrm{z}_{0}=-\infty \\
\frac{\sigma^{2}}{3} z^{3} \text { with } \mathrm{z}_{0}=0
\end{array}\right\} \text { if } a z \text { is small }
$$

More precisely, if $a z$ is small the Taylor series expansion of order 3 gives: 


$$
\sigma_{\Delta_{i}}^{2}(z)=\frac{\sigma^{2}}{2 a^{3}}\left[\left(1-e^{2 a z_{0}}\right)(a z)^{2}+\left(e^{2 a z_{0}}-\frac{1}{3}\right)(a z)^{3}\right]
$$

So it is possible to say that the variance of $\Delta_{i}$ processes can be approximated by a parabolic curve over a range of $a z$ values becoming smaller gradually as $z_{0}$ increases, the interval width vanishing when $z_{0}=0$.

The proposed model allows first to report the measurement results presented in [5], secondly to explain the possibility of evolution law other than parabolic for small $z$ values and ultimately to find the existence of a linear regime for large $z$ values.

It is worth noticing that the transition between these two regimes (small and large values of $a z$ ) can be related to the $z_{\mathrm{c}}=1 / a$ the correlation length of the processes defined from Eq. (10). The measurement of these two regimes allows to estimate the value of the correlation length of the process.

\section{MEASUREMENT SETUPS}

As a transfer operator of the polarization, the Mueller matrix depends on how we define the input and output of the system. Taking into account the averaging process performed by the measurement device is therefore essential. This averaging process does not affect the validity of the Eq. (6). However, it may change the stochastic nature of the $p_{\mathrm{i}}(z)$ processes. This is not the case here for Gaussian property since it is well known that a linear combination of Gaussian processes is still a Gaussian process. Nevertheless, on one hand, that is estimated depends on how we define the input and output and on the other hand, it is obvious that properties of the medium do not depend on the definition of the input and the output. One way to reconcile these remarks is to say that the model used to characterize the medium must be able to account for different ways that input and output of the system may be considered.

In particular, the model must be able to describe the various situations that may occur when the illuminated surface of the sample and the light surface collected at the sample output are variable.

Let us consider first the case of a medium of particles suspended in a liquid (non-stationary samples).

Since we are dealing with homogeneous media, we assert that the two following situations are equivalent - by "equivalent" we means these two configurations lead to the same solution $p(z)$ to Eq. (8) and then the same diagonal entries for of $\log (\mathbf{M})$ -

- Punctually illuminate the medium and collect all the light emerging from the diffusion surface $S_{D}(z)$ at a distance $z$ in the direction collinear to the source-see Fig. 6 (a).

- Illuminate the medium with a collimated source extended on a surface $S_{D}(z)$ and collecting the light emerging from a single point at a distance $z$ in the direction collinear to the source-see Fig. 6 (b).

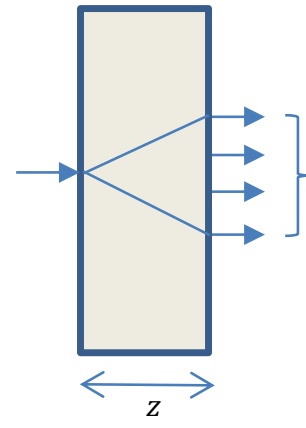

(a)

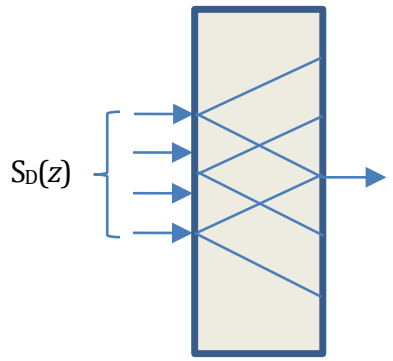

(b)

Fig. 6. Two input-output configurations (a) and (b) leading to the same solution $p(z)$ to Eq. (8) for a homogeneous turbid medium.

The definition of $S_{D}(z)$ can be related to the variance of photon density in the $x-y$ plane at a distance $z$ from the source. It is a function of the corresponding diffusion coefficient [20].

Note that in order to simplify the graphic, the diffusion surface is assumed to vary linearly with $z$ but the results remain valid for any law of variation. Similarly, the surfaces are assumed to be disks and we note $\mathrm{R}_{\mathrm{a}}$ the radius of the disk having a surface $\mathrm{S}_{\mathrm{a}}$.

Consider now the measurement configuration described in Fig. 7 with a collimated source extended on a surface $S_{s}$ and the collected light on a surface $S_{m}$ in a collinear direction of the source. This is the configuration shown in Fig. 1 for instance. Regarding the stochastic process $p(z)$, this configuration can be considered as an integration on the surface $\mathrm{S}_{\mathrm{m}}$ (a spatial summation) of the solution described Fig. 6 (b) provided $R_{\mathrm{s}}$ is greater than $R_{\mathrm{D}}(\mathrm{z})+R_{\mathrm{m}}$. The Central Limit Theorem (CLT) then connects the variance of $p(\mathrm{z})$ in the configuration depicted Fig. 7 with any of the two configurations depicted Fig. 6 since they are equivalent (see Appendix A for the details).:

It is worth noticing that the Gaussian nature of the solution is enhanced by this summation operation in accordance with CLT.

$$
\operatorname{Var}\left[p(z) / R_{m}\right]=\left(1+\frac{R_{m}}{R_{D}(z)}\right) \operatorname{Var}\left[p(z) / R_{D}(z)\right]
$$




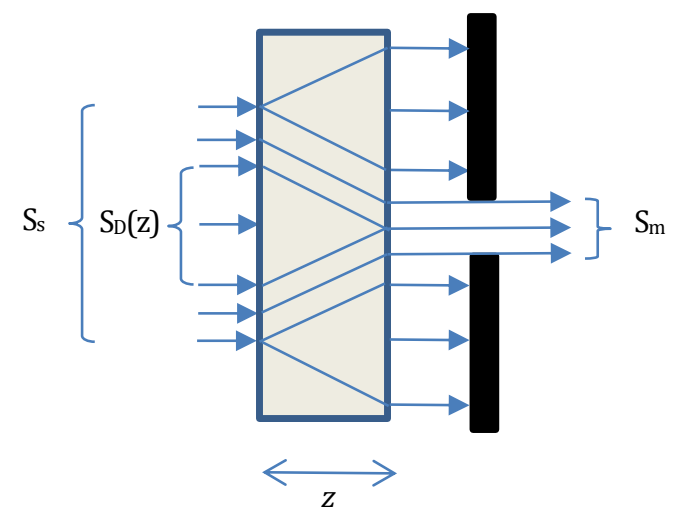

Fig. 7. Measurement configuration with a collimated source extended on a surface $S_{s}$ and a collected light on a surface $S_{m}$ in a collinear direction of the source

Two other equivalent situations are depicted Fig. 8.

- A collimated extended source and a cone of collected light with respect to one output point- see Fig. 8 (a) - Generally, a lens with a numerical aperture $N A$ collimates the collected light towards the sensor.

- An input cone of light with a maximal angle corresponding to the preceding $N A$ and a collimated output- see Fig. 8 (b).

To determine the expression of $p(z)$ for this new measurement configuration, it is worth recalling the general form of the solution [17] of Eq. (8):

$$
p(z)=e^{-a\left(z-z_{0}\right)} p_{0}+\int_{z_{0}}^{z} \sigma e^{a(u-z)} d B(u)
$$

where $p_{0}$ is the initial condition when $z=z_{0}$. For the previous configurations shown Fig. 6 and Fig. 7 where input and output are collinear, $p_{0}$ is a constant term. If $z_{0}$ is the coordinates of the input surface of the medium, we obviously have $p_{0}=0$.

In the case of the configuration described Fig. 8 (b) (and thus in that of Fig. 8 (a) as they are equivalent), $p_{0}$ becomes a random variable. Now is the average value of $p_{0}$ which is zero (keeping the same convention for $z_{0}$ ) and its variance $\operatorname{Var}\left(p_{0}\right)$ is directly related to the value of the numerical aperture $N A$. The two previous cases can also be considered as the limit when $N A$ vanishes since a constant is a random variable with null variance.

From Appendix B a new expression of $p$ covariance is given by:

$$
\begin{aligned}
& \operatorname{Cov}\left[p\left(z_{1}\right) p\left(z_{2}\right) / z_{0}\right]= \\
& e^{-a\left(z_{2}+z_{1}-2 z_{0}\right)}\left[\operatorname{Var}\left(p_{0}\right)-\frac{\sigma^{2}}{2 a}\right]+\frac{\sigma^{2}}{2 a} e^{-a\left(z_{2}-z_{1}\right)}
\end{aligned}
$$

It is straightforward to see that if $\operatorname{Var}\left(p_{0}\right)=0$ we find the expression of Eq. (10) leading to the solution presented Eq. (12) for the variance of $\Delta_{i}$ processes. In the same way, the maximum value of the variance is reached when the cone of light - and therefore $N A=N A_{\max }$ - is sufficient to capture all output beams. Then we have $\operatorname{Var}\left(p_{0}\right)=\sigma^{2} / 2 a$ and the covariance is $\mathrm{z}_{0}$ independent leading to the stationary solution of Eq. (13) when $\mathrm{z}_{0}=-\infty$. If $N A$ is changing from 0 to $N A_{\max } \operatorname{Var}\left(p_{0}\right)$ changes from 0 to $\sigma^{2} / 2 a$ and the variance of $\Delta_{i}$ processes is changing continuously from a non-stationary solution to a stationary solution. A straightforward calculation (see Appendix B) shows that the set of solutions obtained for the variance of $\Delta_{i}$ processes when $\operatorname{Var}\left(p_{0}\right)$ is changing from 0 to $\sigma^{2} / 2 a$ is the same as those shown in Eq. (12) when $z_{0}$ is changing from 0 to $-\infty$.

In the case of mounting with an output lens, the validity of the parabolic curve zone will therefore be directly related to the numerical aperture of the lens since changing the numerical aperture is equivalent to changing the value of $\operatorname{Var}\left(p_{0}\right)$ or the $z_{0}$ value in Eq. (15) as previously explained in Section 3-B. From an experimental point of view, it is not necessary to know a priori the relation between $N A$ and $\operatorname{Var}\left(p_{0}\right)$. zo may indeed be considered as an additional parameter of Eq. (12) to estimate as well as $a$ and $\sigma$.

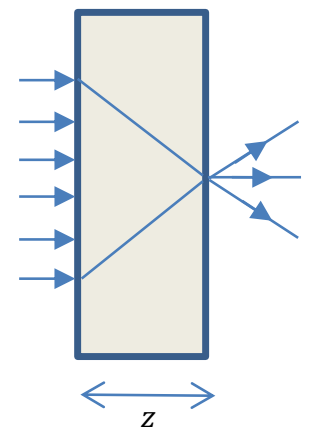

(a)

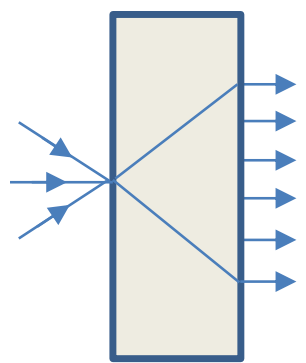

(b) 
The proposed model used to characterize the medium is then able to account for different ways that input and output of the system may be considered. Fig. 9-10 show the experimental results obtained for the different measurement setups. The sample consisted of diluted milk with a dilution $=0.13$ strong enough so as to have the non-linear zone for not too small $z$ values. Only the non-linear variation area (small $a z$ values) is represented as it is what distinguishes the different regimes of variations: parabolic, cubic or intermediate. The experimental values are obtained in the case of Fig. 9 from the setup presented in Fig.1. We are therefore in the case of a measurement setup described Fig. 7. The results clearly show an evolution process in $z^{3}$. Fig. 10 is obtained from the setup presented in Fig.1 but only in collecting the light from a point on the exit surface by a lens with focal length $f=140 \mathrm{~mm}$ placed before the PSA. We are therefore in the case of a measurement configuration described Fig. 8 (a). The results clearly show that a Taylor series expansion of order 3 (see Eq. (15)) is now needed to properly describe the evolution of the logarithmic depolarization.

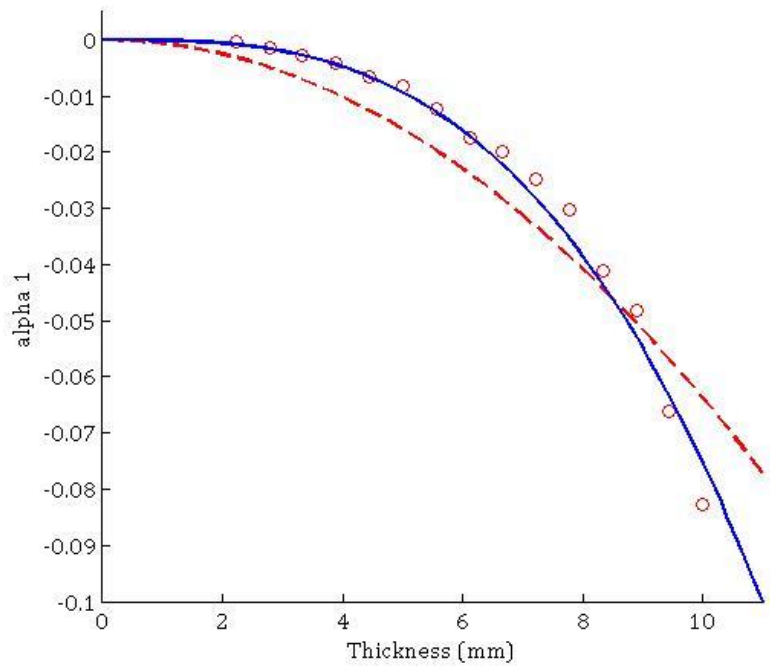

Fig. 9. Evolution of the logarithmic depolarizations $\alpha_{1}$ with $z$ the thickness of medium traveled by light for a dilutions $d=0.13$ of milk. o are experimental values measured with the measurement setup depicted Fig. 1. Solid line and dashed line are respectively the best cubic and parabolic fit.

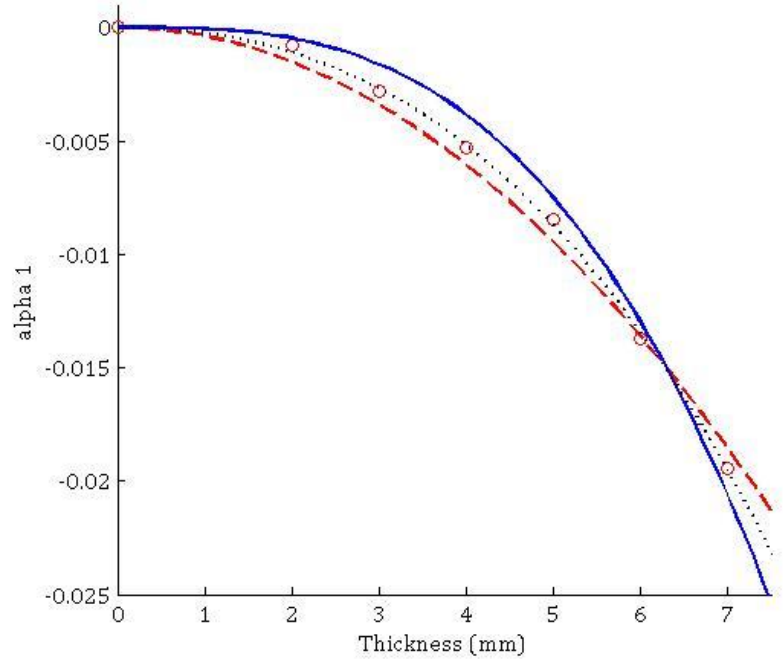

Fig. 10. Evolution of the logarithmic depolarizations $\alpha_{1}$ with $z$ the thickness of medium traveled by light for a dilutions $d=0.13$ of milk. o are experimental values. Light is collected by a lens (focal length $f=140 \mathrm{~mm}$ ). Solid line and dashed line are respectively the best cubic and parabolic fit. The dotted line is derived from Eq. (12).

Let us now consider the case of a solid medium with static scatterers (stationary samples). As the process is ergodic, the statistical moments can be obtained by computing the moments on all the possible events of the $p(z)$ process. The spatial averaging that is mandatory in this case assumes the use of an extended source (collimated or not). Such a source therefore increases the number $N$ of events of the $p(z)$ process involved in the moments estimation. Each element of the source provides a realization of the $p(z)$ process that encounters an initial value $p_{0}$ which depends on the input point in the medium and is therefore a random variable. We thus find the stationary configuration for $p(z)$ obtained when the initial condition is random with a maximum variance if the number of realizations of the process collected by the sensor is sufficient. 
Increasing the width of the source can be also treated by the strong law of large numbers as in the case of non-stationary environments but now applied to the problem of estimating the parameters of a probability distribution.

Fluctuations around the theoretical average after $N$ experiments are of the order $\sigma / \sqrt{N}$ (see Appendix A). Increasing the thickness of the medium leads to fewer recovered statistical events at the exit point, due to the diffusive behavior of the medium. So, the variance of the estimate will increase as the thickness of the medium increases. This is clearly visible in the results presented in Fig. 2 of [5] where the values of the dispersion of measurement increase sharply when $z$ increases. This phenomenon is obviously not observed in the case of non-stationary environments since the stochastic nature is completely described by a single temporal realization. It is worth noticing that increasing the power of the source does not change the problem from the $p(z)$ estimation point of view. A solution to avoid increasingly strong fluctuations, would be to extend the measurement surface as $z$ increases.

\section{MEASUREMENT AND PARAMETER ESTIMATION}

\section{A. Estimation of spectroscopic quantities.}

From an experimental point of view, we only access to the measurement of $\log (\mathbf{M})$ entries and Eq. (6) gives the following relations:

$$
\left\{\begin{array}{l}
\alpha_{1}=-\left(\sigma_{\Delta_{2}}^{2}+\sigma_{\Delta_{5}}^{2}\right)-\left(\sigma_{\Delta_{3}}^{2}+\sigma_{\Delta_{6}}^{2}\right) \\
\alpha_{2}=-\left(\sigma_{\Delta_{1}}^{2}+\sigma_{\Delta_{4}}^{2}\right)-\left(\sigma_{\Delta_{3}}^{2}+\sigma_{\Delta_{6}}^{2}\right) \\
\alpha_{3}=-\left(\sigma_{\Delta_{1}}^{2}+\sigma_{\Delta_{4}}^{2}\right)-\left(\sigma_{\Delta_{2}}^{2}+\sigma_{\Delta_{5}}^{2}\right)
\end{array}\right.
$$

Note that the upper diagonal entry has been subtracted from the main diagonal in order to find the same expression for M as in Eq. (3). This is always possible since differential matrix is defined within an additive identity matrix multiplied by an arbitrary constant [15].

Eq. (19) shows that it is therefore not possible to estimate the behavior of the six $\sigma_{\Delta}$ parameters separately. It is further obvious that switching between index 1 and 4 ( 2 and 5 or 3 and 6 respectively) would not change the measurement results. This comment reflects the fact that it is actually possible to access only three quantities:

$$
\left\{\begin{array}{c}
V_{L}=\left(\sigma_{\Delta_{1}}^{2}+\sigma_{\Delta_{4}}^{2}\right) \\
V_{L 45}=\left(\sigma_{\Delta_{2}}^{2}+\sigma_{\Delta_{5}}^{2}\right) \\
V_{C}=\left(\sigma_{\Delta_{3}}^{2}+\sigma_{\Delta_{6}}^{2}\right)
\end{array}\right.
$$

where $V_{\text {sp }}$ (with $\left.s p \in\{\mathrm{L}, \mathrm{L} 45, \mathrm{C}\}\right)$ stands for the variance of $s p$ quantity. $\mathrm{L}, \mathrm{L} 45$ and C are defined in the spectroscopic notation by $\mathrm{L}=-\left(p_{1}+\mathrm{i} . p_{4}\right), \mathrm{L} 45=-$ $\left(p_{2}+\mathrm{i} . p_{5}\right)$ and $\mathrm{C}=\left(p_{3}+\mathrm{i} . p_{6}\right)$. This remark can also be directly related to the expression of the equivalent reduced coherency matrices termed $\mathbf{C} 3$ defined [19] by the following relations (for the diagonal matrices $\mathbf{m}$ ):

$$
\mathbf{C 3}=\left[\begin{array}{ccc}
\mathrm{V}_{\mathrm{L}} & 0 & 0 \\
0 & \mathrm{~V}_{\mathrm{L} 45} & 0 \\
0 & 0 & \mathrm{~V}_{\mathrm{C}}
\end{array}\right] \text { with }\left\{\begin{array}{l}
\mathrm{V}_{\mathrm{L}}=\frac{\alpha_{3}+\alpha_{2}-\alpha_{1}}{2} \\
\mathrm{~V}_{\mathrm{L} 45}=\frac{\alpha_{3}+\alpha_{1}-\alpha_{2}}{2} \\
\mathrm{~V}_{\mathrm{C}}=\frac{\alpha_{1}+\alpha_{2}-\alpha_{3}}{2}
\end{array}\right.
$$

Measurements of $\alpha_{\mathrm{i}}$ therefore allow to estimate the $\left(a_{\mathrm{sp}}, \sigma_{\mathrm{sp}}\right)$ parameters of stochastic models of L, L45 and C from the relationships given Eq. (21).

The lines drawn in Fig. 2 for the full cream milk and Fig. 3 for the half cream milk are obtained by injecting these parameter values in the nonstationary model of the Eq. (13) for each of L, L45 and C quantities. The $\alpha_{\mathrm{i}}$ curves are then obtained by inverting the relationship of Eq. (21).

\section{B. Parameters relation to dilution}

Since for large $z$ values, $\sigma_{\Delta_{i}}^{2}$ is linear the corresponding straight line is characterized - see Eq. (13) - by a slope $S=(\sigma / a)^{2}$ and "vertical axis intercept" $b=-3 \sigma^{2} /\left(2 a^{3}\right)$ or $b=-\sigma^{2} / a^{3}$ for non-stationary and stationary media respectively. The $(a, \sigma)$ parameters can thus be estimated from the two ratios $S / b$ and $S^{3 / 2} / b$.

An example of the estimated values for the three respective dilutions $d$ are given Table 1 from the measurements of Fig. 2 .

It is worth noticing that:

a) - Estimated $S$ values show a linear dependence to $d$,

b) - From [5] the parabolic coefficient is proportional to $d^{2}$. Obviously the parabolic coefficients of the variances of L, L45 and C models inherit this property. 
Table 1. Slope $S$ and vertical axis intercept $b$ for the three respective dilutions $d$ of Fig. 2.

\begin{tabular}{lllllll}
\hline $\mathrm{d}$ & 0.048 & & 0.095 & & 0.167 & \\
\hline & $\mathrm{S}$ & $\mathrm{b}$ & $\mathrm{S}$ & $\mathrm{b}$ & $\mathrm{S}$ & $\mathrm{b}$ \\
\hline $\mathrm{L}$ & 0.084 & 0.12 & 0.169 & 0.158 & 0.296 & 0.117 \\
$\mathrm{~L} 45$ & 0.084 & 0.119 & 0.169 & 0.155 & 0.295 & 0.109 \\
$\mathrm{C}$ & 0.117 & 0.343 & 0.237 & 0.344 & 0.371 & 0.237 \\
\hline
\end{tabular}

Consequently one can deduce that $a$ and $\sigma^{2}$ are respectively proportional to $d$ and $d^{3}$. Confirmation of these results is shown Fig. 11-12 where the evolution of the model parameters $a$ and $\sigma^{2 / 3}$ with the dilution are presented.

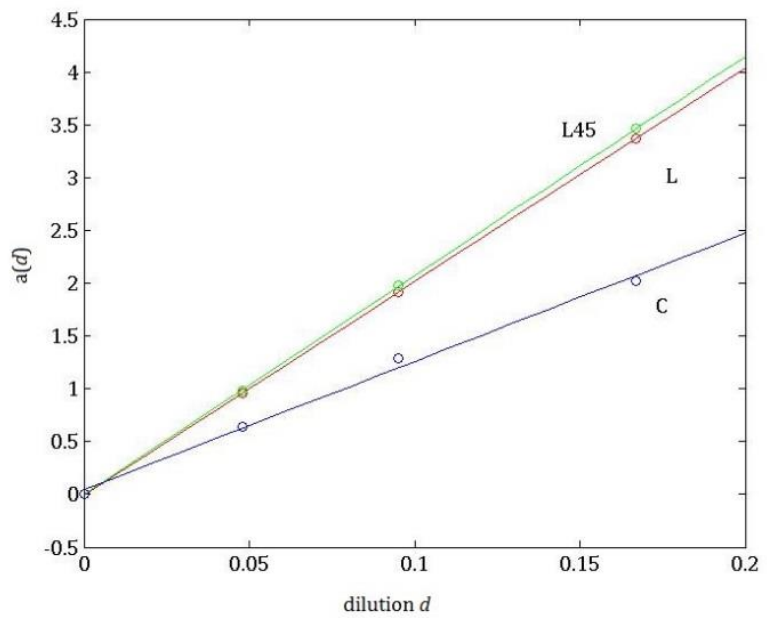

Fig. 11. Evolution of the model parameters $a_{\mathrm{sp}}-s p \in\{\mathrm{L}, \mathrm{L} 45, \mathrm{C}\}$ - with the dilution $d . o$ are estimated values. The solid lines are linear fits to the experimental data.

The ways in which $a$ and $\sigma$ depend on $d$ also lead to the consequence that $b$ should be independent of the dilution $d$. Assuming that the variations observed on $b$ are due to measurement accuracy, estimation of $a$ and $\sigma$ parameters were made by using the average value of $b$ for the three dilutions.

$\sigma$ is related to the noise magnitude and it seems credible that the noise level physically caused by the scattering events is a function of the concentration of particles. Since $a$ is proportional to the dilution, $z_{\mathrm{c}}=1 / a$ the correlation length of the random processes $p_{\mathrm{i}}(z)$ is thus -see Eq. (10) inversely proportional to the concentration of particles. The higher the concentration increases faster the un-correlation appears.

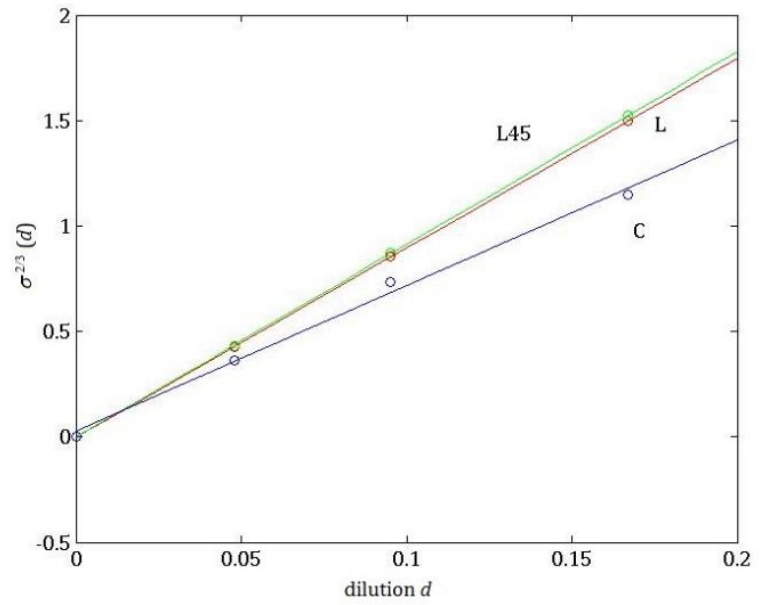

Fig. 12. Evolution of the model parameters $\sigma_{s p}{ }^{2 / 3}-s p \in\{L, L 45, C\}$ - with the dilution $d . o$ are estimated values. The solid lines are linear fits to the experimental data.

Table 2 gives $a$ and $\sigma$ estimated parameters for the measurements presented Fig. 3. It is straightforward to verify that the laws of evolution of these parameters with the dilution previously announced are still valid. 


\begin{tabular}{ccccc}
\hline $\mathrm{d}$ & \multicolumn{2}{c}{0.286} & \multicolumn{2}{c}{0.429} \\
\hline & $\mathrm{a}$ & $\sigma$ & $\mathrm{a}$ & $\sigma$ \\
\hline $\mathrm{L}$ & 0.189 & 0.101 & 0.261 & 0.164 \\
$\mathrm{~L} 45$ & 0.189 & 0.101 & 0.261 & 0.164 \\
$\mathrm{C}$ & 0.22 & 0.108 & 0.319 & 0.191 \\
\hline
\end{tabular}

\section{Bi-dimensional model for spectroscopic quantities}

The model proposed Eq. (13) thus allows very precise adjustments to experimental data presented Fig. 2 and Fig. 3. However it is not sufficient to account for the evolution of the $\alpha_{3}$ term observed in the case of measurements presented Fig. 5.

In the above reasoning, we have assumed that the stochastic processes were one-dimensional. The reason for this assumption is that the differential matrix is diagonal. From Eq. (5) limited to the second order cumulants, it is possible [3] to write the depolarizing part of matrix $\mathbf{m}$ in the general form:

$$
\left[\begin{array}{cccc}
\sigma_{4}^{2}+\sigma_{5}^{2}+\sigma_{6}^{2} & -\sigma_{26}+\sigma_{35} & -\sigma_{34}+\sigma_{16} & -\sigma_{15}+\sigma_{24} \\
\sigma_{26}-\sigma_{35} & \sigma_{4}^{2}-\sigma_{2}^{2}-\sigma_{3}^{2} & \sigma_{12}+\sigma_{45} & \sigma_{13}+\sigma_{46} \\
\sigma_{34}-\sigma_{16} & \sigma_{12}+\sigma_{45} & \sigma_{5}^{2}-\sigma_{1}^{2}-\sigma_{3}^{2} & \sigma_{23}+\sigma_{56} \\
\sigma_{15}-\sigma_{24} & \sigma_{13}+\sigma_{46} & \sigma_{23}+\sigma_{56} & \sigma_{6}^{2}-\sigma_{1}^{2}-\sigma_{2}^{2}
\end{array}\right]
$$

where $\sigma_{i j}$ denotes the entries of the covariance matrix of the process $\Delta_{i}(z)$ ( $\sigma_{i i}$ will be denoted by $\sigma^{2}$ and is the variance of the process).

The measured matrices are diagonal, this imposes null covariance terms between processes. It is worth noticing that all combinations of covariance terms between processes are not present in the given expression of Eq. (22). The covariance terms between processes 1 and 4,2 and 5 or 3 and 6 respectively are missing. This is obviously the terms of covariance between components (real and imaginary part) of the three spectroscopic parameters L, L45 and C. It is therefore possible to have a non-zero covariance for these components without affecting the diagonal nature of the differential matrix. Any other combination is impossible because it shows an off diagonal term. From the standpoint of physical phenomena, it means (see the definition of spectroscopic parameters L, L45 and C) that there are relationships between similar quantities of birefringence and dichroism (linear birefringence and linear dichroism and so on). It is therefore justified to regard the components of process L, L45 and C as two-dimensional processes described by a differential equation of the form:

$$
\left(\begin{array}{c}
d p_{i} \\
d p_{i+3}
\end{array}\right)=\mathbf{A}_{\mathbf{i}}\left(\begin{array}{c}
p_{i} \\
p_{i+3}
\end{array}\right)+\mathbf{S}_{\mathbf{i}}\left(\begin{array}{c}
d B_{i} \\
d B_{i+3}
\end{array}\right)
$$

where i $\in\{1,2,3\}, \mathbf{A}_{i}$ and $\mathbf{S}_{i}$ are $2 \times 2$ matrices with real entries. Without confusion $\mathbf{A}_{i}$ and $\boldsymbol{S}_{\mathrm{i}}$ will be denoted $\mathbf{A}$ and $\mathbf{S}$ in the following to simplify notation.

Since $\mathbf{A}$ has real entries, its eigenvalues are either real or complex conjugates. $\mathbf{A}$ and $\mathbf{S}$ can therefore be decomposed as follows:

$$
\mathbf{A}=\mathbf{L} \Theta \mathbf{L}^{-1} \quad \mathbf{V}=\mathbf{L}^{-1} \mathbf{S}
$$

where $\Theta$ is a diagonal matrix and $\mathbf{L}$ is obtained from the eigenvectors of $\mathbf{A}$. According to the real or complex nature of the eigenvalues, the $\Theta$ matrix has one of the following expressions

$$
\Theta_{1}=\left(\begin{array}{cc}
-\lambda_{1} & 0 \\
0 & -\lambda_{2}
\end{array}\right) \quad \Theta_{2}=\left(\begin{array}{cc}
-\gamma & -\omega \\
\omega & -\gamma
\end{array}\right)
$$

A new set of processes can be defined by $\mathbf{X}=\mathbf{L}^{-1} \mathbf{P}$ where $\mathbf{P}=\left(p_{\mathrm{i}} p_{\mathrm{i}+3}\right)^{\mathrm{T}}\left(\mathrm{x}^{\mathrm{T}}\right.$ stands for the transpose of vector or matrix $)$. These processes are driven by the following stochastic differential equation:

$$
d \mathbf{X}(z)=\Theta \mathbf{X}(z) d z+\Sigma d B(z)
$$

where $\Sigma=\mathbf{V} \mathbf{V}^{\mathrm{T}}$. Eq. (22) shows that $\mathbf{X}$ vector is a two-dimensional $O U$ process. The covariance matrix of $\mathbf{X}$ vector at $z_{1}$ and $z_{2}$ related to $z_{0}$ is given by:

$$
\begin{aligned}
& \operatorname{Cov}\left(\mathbf{X}\left(z_{1}\right) \mathbf{X}\left(z_{2}\right)^{\mathrm{T}} / z_{0}\right)=\int_{z_{0}}^{z_{1}} e^{\Theta\left(u-z_{1}\right)} \Sigma e^{\Theta\left(u-z_{2}\right)} d u \\
& z_{0} \leq z_{1} \leq z_{2}
\end{aligned}
$$

When $\Theta$ has $\Theta_{1}$ expression it is straightforward to demonstrate that Eq. (27) leads to z-evolution of the processes identical to those of Eq. (13) for the stationary $\left(\mathrm{z}_{0} \rightarrow-\infty\right)$ and non-stationary $\left(\mathrm{z}_{0}=0\right)$ cases. When $\Theta$ has $\Theta_{2}$ expression, upon substituting Eq. (27) into the computation of variance of the processes and a bit of algebra, we find expressions given Eq. (28) for the non-stationary solution ( $s p \in\{L, L 45, C\}$ when $i \in\{1,2,3\}$ respectively): 


$$
\begin{aligned}
\sigma_{s p}^{2} & =\sigma_{i}^{2}+\sigma_{i+3}^{2} \\
& =B_{i} z+\left[D_{i} \cos \left(\omega_{i} z\right)+E_{i} \sin \left(\omega_{i} z\right)\right] e^{-\gamma_{i} z}+F_{i} e^{-2 \gamma_{i} z}+K_{i}
\end{aligned}
$$

and given Eq. (29) for the stationary solution:

$$
\begin{aligned}
\sigma_{s p}^{2} & =\sigma_{i}^{2}+\sigma_{i+3}^{2} \\
& =B_{i}^{\prime} z+\left[D_{i}^{\prime} \cos \left(\omega_{i} z\right)+E_{i}^{\prime} \sin \left(\omega_{i} z\right)\right] e^{-\gamma_{i} z}+K_{i}^{\prime}
\end{aligned}
$$

$\left\{B_{\mathrm{i}}, D_{\mathrm{i}}, \ldots, K_{\mathrm{i}}\right\}$ and $\left\{B_{\mathrm{i}}, D_{\mathrm{i}}^{\prime}, \ldots, K_{\mathrm{i}}\right\}$ are functions of the parameters $\left\{\gamma_{\mathrm{i}}, \omega_{\mathrm{i}}, \mathbf{S}\right\}$. The analytical expression of these factors is not usable, but they satisfy the following relationships that achieve a first non-zero term of $z^{3}$ order $\left(z^{2}\right.$ order respectively) for expansion in power of $\sigma_{s p}$ for small $z$ value:

$$
\begin{gathered}
\left\{\begin{array}{c}
K+F+D=0 \\
B-2 \gamma F-\gamma D+E \omega=0 \\
4 \gamma^{2} F+D\left(\gamma^{2}-\omega^{2}\right)-2 \gamma \omega E=0
\end{array}\right. \\
\left\{\begin{array}{c}
K^{\prime}+D^{\prime}=0 \\
B^{\prime}-\gamma D^{\prime}+E^{\prime} \omega=0
\end{array}\right.
\end{gathered}
$$

Table 3 gives estimated parameters for the measurements presented Fig. 4 and Fig. 5.

Table 3. Parameter estimation of L, L45 and C for the dilution $d=0.276$ of Fig. 4 and Fig. 5

\begin{tabular}{lccccccl}
\hline \multicolumn{1}{c}{$s p$} & $\gamma$ & $\omega$ & $\mathrm{B}$ & $\mathrm{K}$ & $\mathrm{D}$ & $\mathrm{E}$ & $\mathrm{F}$ \\
\hline $\mathrm{L}$ & 0.126 & 0.47 & 0.09 & -0.018 & 0.026 & 0.018 & 0.007 \\
$\mathrm{~L} 45$ & 0.126 & 0.47 & 0.09 & -0.023 & 0.023 & 0.017 & $10^{-4}$ \\
$\mathrm{C}$ & 0.086 & 0.14 & 0.02 & -0.12 & 0.049 & 0.037 & 0.062 \\
\hline
\end{tabular}

Fig. 13 shows the resulting curves obtained by injecting these parameter values in the non-stationary model of the Eq. (28) for each of L, L45 and C quantities. As previously, $\alpha_{i}$ curves of Fig. 4 and Fig. 5 are obtained by inverting the relationship of Eq. (21).

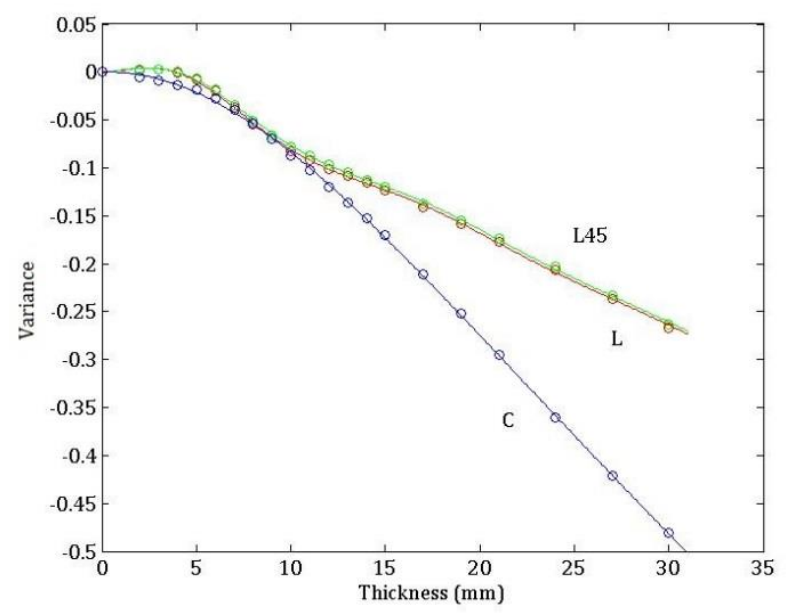

Fig. 13. Evolution of the $\sigma_{L, L 45, C}^{2}$ with $z$ the thickness of medium traveled by light for dilution $d=0.276$ of pastis. o are experimental values. The solid line are derived from Eq. (28).

First, the model proposed Eq. (28)-(29) confirms its ability to very precise adjustments to experimental data and secondly the existence of weak correlation between $p_{\mathrm{i}}$ and $p_{\mathrm{i}+3}$ variables in the case of this medium. The cause of these correlations has yet to be identified. However, the proposed model is able to account for these effects. It should be noted that this approach also provides the method to handle the case of non-diagonal Mueller matrix where the off-diagonal terms are covariance coefficients between processes.

\section{CONCLUSION}

In summary, we have described the existence of different operating zones, in spatial evolution of depolarization in homogeneous turbid media. We show the existence of different regimes in spatial evolution of depolarization in turbid media characterized by a diagonal Mueller matrix (pure depolarizer). Experimental results previously published [5], already established the existence of a first regime where the depolarization follows a parabolic law with the thickness of the medium traveled by light. New experiments firstly confirm the existence of a second regime which we have previously demonstrated [3] where the depolarization follows a linear law on a large scale. They also confirm the existence of much more complex evolution laws even under small-scale approximation. A stochastic approach is proposed to model the phenomenon in the case of solid scattering 
media (stationary hypothesis) and liquid scattering media (non-stationary hypothesis). It perfectly describes all these different experimental results and allows to analyze the behavior of the polarization in the case of solid or liquid scattering media. The influence of the measurement setup is also analyzed. The approach notably allows to analyze the transition between the different regimes in terms of correlation of the fluctuations that are associated with the existence of the depolarization. The model parameters are straightforwardly related to particles concentration (or dilution for the liquids). The analysis of the effect of particle size on these parameters and on the boundaries of the validity of the model is obviously a possible continuation of this work.

We believe that these results will find their applications in areas such as biological tissues or cloud of particles analysis for instance.

\section{APPENDIX A}

According to the Central Limit Theorem, when $N$ independent and identically distributed random variables (with mean value given by $m$ and variance by $\sigma^{2}$ ) are added, their sum $S_{N}$ converges in distribution to a normal distribution. Formally, this theorem can be stated as follows:

$$
\frac{S_{N}-N m}{\sigma \sqrt{N}} \rightarrow \mathcal{N}(0,1)
$$

For $p(z)$ process (with $m=0$ and $\sigma^{2}=\operatorname{Var}[p(z)]$ ) integrated on a surface $\mathrm{S} 1$, we then have:

$$
\frac{\int_{S 1} p(z) d s}{S 1} \approx \frac{\sqrt{\operatorname{Var}[p(z)]}}{\sqrt{S 1}} \mathcal{N}(0,1)
$$

Consequently, the variance of the $\int_{S 1} p(z) d s$ process is given by $\operatorname{Var}[p(z) / \mathrm{S} 1]=\mathrm{S} 1 . \operatorname{Var}[p(z)]$ and we have the following relationship:

$$
\frac{\operatorname{Var}[p(z) / S 1]}{S 1}=\frac{\operatorname{Var}[p(z) / S 2]}{S 2}
$$

If the diffusion surfaces are assumed to be disks and we denote $A$ and $B$ the radius of the two disks - one on the left side and one on the right side respectively (see Fig. A1) - we have the following relation for the variance of $p$ :

$$
\begin{aligned}
\operatorname{Var}[p(z) / B] & =\frac{\pi(A+B)^{2}}{\pi A^{2}} \operatorname{Var}[p(z) / B=0] \\
& =\left(1+\frac{B}{A}\right)^{2} \operatorname{Var}[p(z) / B=0]
\end{aligned}
$$

(A4)

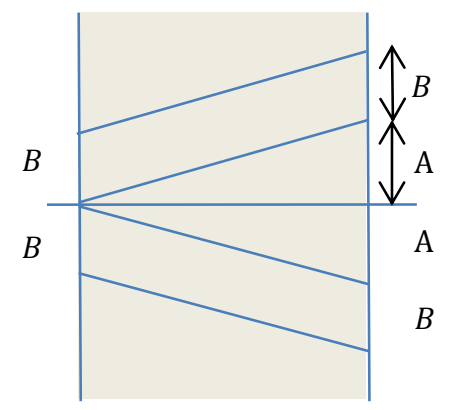

Fig. A1 Schematic representation of the evolution of the diffusion surface when passing through the medium.

\section{APPENDIX B}

Solution of the equation:

$$
\begin{aligned}
& d X(z)=-a X(z) d z+\sigma d B(z) \\
& \text { with } X\left(z_{0}\right)=X_{0}
\end{aligned}
$$

Is given by [17]:

$$
X(z)=e^{-a\left(z-z_{0}\right)} X_{0}+\int_{z_{0}}^{z} \sigma e^{a(u-z)} d B(u)
$$


The second order moment of the process considered at $z_{1}$ and $z_{2}$ is given by (with $z_{0}<z_{1}<z_{2}$ ):

$$
\begin{aligned}
& E\left[X\left(z_{1}\right) X\left(z_{2}\right)\right]=e^{-a\left(z_{1}+z_{2}-2 z_{0}\right)} E\left[X_{0}^{2}\right]+I \\
& \text { with } I=E\left[\int_{z_{0}}^{z_{1}} \sigma e^{a\left(u-z_{1}\right)} d B(u) \int_{z_{0}}^{z_{2}} \sigma e^{a\left(v-z_{1}\right)} d B(v)\right]
\end{aligned}
$$

Using the property of the uncorrelation of Brownian process B on disjoint intervals and the Itô isometry, this integral can be written as:

$$
\begin{aligned}
& I=E\left[\int_{z_{0}}^{z_{1}}\left(\sigma e^{a\left(u-z_{1}\right)}\right)^{2} d u\right] e^{-a\left(z_{2}-z_{1}\right)} \\
& I=\frac{\sigma^{2}}{2 a}\left[e^{-a\left(z_{2}-z_{1}\right)}-e^{-a\left(z_{2}+z_{1}-2 z_{0}\right)}\right]
\end{aligned}
$$

Eq. (B3) and Eq. (B4) gives the following expression for the covariance of $X$ process considered at $z_{1}$ and $z_{2}$ :

$$
\begin{aligned}
& \operatorname{Cov}\left[X\left(z_{1}\right) X\left(z_{2}\right) / z_{0}\right]= \\
& e^{-a\left(z_{2}+z_{1}-2 z_{0}\right)}\left[\operatorname{Var}\left(X_{0}\right)-\frac{\sigma^{2}}{2 a}\right]+\frac{\sigma^{2}}{2 a} e^{-a\left(z_{2}-z_{1}\right)}
\end{aligned}
$$

We can then define a variable denoted by $\mathrm{z}_{\alpha}$ as:

$$
\begin{aligned}
& \operatorname{Var}\left(X_{0}\right)=\alpha \frac{\sigma^{2}}{2 a} \text { with } 0 \leq \alpha \leq 1 \\
& z_{\alpha}=\frac{1}{2 a} \log (1-\alpha) \text { with }-\infty<z_{\alpha} \leq 0
\end{aligned}
$$

We have:

$$
\operatorname{Cov}\left[X\left(z_{1}\right) X\left(z_{2}\right) / z_{0}\right]=\frac{\sigma^{2} e^{-a\left(z_{2}-z_{1}\right)}}{2 a}\left[1-e^{-2 a\left[z_{1}-\left(z_{0}+z_{\alpha}\right)\right]}\right]
$$

If we set $z_{0}=z_{0}+z_{0}$, we find exactly the expression of Eq. (10) provided to replace $z_{0}$ by $z_{0}$. If $z_{0}$ is the coordinates of the input surface of the medium $\left.\left.\left(z_{0}=0\right), z_{0} \in\right]-\infty, 0\right]$.

\section{References}

1. R. M. A. Azzam, "Propagation of partially polarized light through anisotropic media with or without depolarization: A differential $4 \times 4$ matrix calculus," J. Opt. Soc. Am. 68, 1756-1767 (1978)

2. V. Devlaminck, "Physical model of differential Mueller matrix for depolarizing uniform media," J. Opt. Soc. Am. 30, 2196-2204 (2013)

3. V. Devlaminck, "Depolarizing differential Mueller matrix of homogeneous media under Gaussian fluctuation hypothesis," J. Opt. Soc. Am. A 32, 1736-1743 (2015).

4. R. Ossikovski, O. Arteaga, "Statistical meaning of the differential Mueller matrix of depolarizing homogeneous media," Opt. Letters. 39, 4470-4473 (2014)

5. N. Agarwal, J. Yoon, E. Garcia-Caurel, T. Novikova, J. Vanel, A. Pierangelo, A. Bykov, A. Popov, I. Meglinski, and R. Ossikovski, "Spatial evolution of depolarization in homogeneous turbid media within the differential Mueller matrix formalism," Opt. Lett. 40, 5634-5637 (2015).

6. J. Bueno, E. Berrio, M. Ozolinsh, and P. Artal, "Degree of polarization as an objective method of estimating scattering," J. Opt. Soc. Am. A 21, 1316-1321 (2004).

7. M. Li, P. Lu, Z. Yu, L. Yan, Z. Chen, C. Yang, and X. Luo, "Vector Monte Carlo simulations on atmospheric scattering of polarization qubits," J. Opt. Soc. Am. A 30, 448-454 (2013).

8. S. Otsuki, "Symmetry relationships for multiple scattering of polarized light in turbid spherical samples: theory and a Monte Carlo simulation," J. Opt. Soc. Am. A 33, 258-269 (2016).

9. B. Kunnen, C. Macdonald, A. Doronin, S. Jacques, M. Eccles, and I.Meglinski, "Application of circularly polarized light for non-invasive diagnosis of cancerous tissues and turbid tissue-like scattering media," J. Biophotonics 8, 317-323 (2015).

10. A. Pierangelo, A. Benali, M. Antonelli, T. Novikova, P. Validire, B. Gayet, and A. De Martino, "Ex-vivo characterization of human colon cancer by Mueller polarimetric imaging," Opt. Express 19, 1582-1593 (2011).

11. H. C. van de Hulst, Light Scattering by Small Particles (Dover, 1981).

12. G. Puentes, D. Voigt, A. Aiello, and J. Woerdman, "Experimental observation of universality in depolarized light scattering," Opt. Lett. 30, 3216-3218 (2005)

13. R.F. Fox, "Gaussian stochastic processes in physics," Phy. Rep. 48, 179-283 (1978)

14. N.G. van Kampen, Stochastic processes in physics and chemistry (North Holland Publishing Company, 1981)

15. R. Ossikovski, "Differential matrix formalism for depolarizing anisotropic media," Opt. Letters. 36, 2330-2332 (2011)

16. G. E. Uhlenbeck and L. S. Ornstein, "On the theory of the Brownian motion," Phy. Rev. 36, 823-841 (1930) 
17. Bert Oksendal, Stochastic differential equations ( Springer-Verlag Berlin Heidelberg, 1985)

18. R. Furth, "The Brownian motion with consideration of the longevity of the direction of movement," Zeits. f. Physik 2, 244-256 (1920)

19. R. Ossikovski, V. Devlaminck, "General criterion for the physical realizability of the differential Mueller matrix," Opt. Lett. 39, 1216-1219 (2014)

20. W. Cai, M. Lax, and R. R. Alfano, “Cumulant solution of the elastic Boltzmann transport equation in an infinite uniform medium,”Phy. Rev. E 61, 3871-3876 (2000). 